\title{
Estudo sobre elicitação de requisitos para o desenvolvimento de aplicativos móveis
}

\author{
${ }^{1}$ Ramon W. S. Fonseca, ${ }^{1}$ Mariana de A. Falcão, ${ }^{1}$ Welliana B. Ramalho, ${ }^{1}$ Joêmia \\ Leilane Gomes de Medeiros \\ 1Universidade Federal Rural do Semi-árido (UFERSA) \\ R. Gamaliel Martins Bezerra, 59515-000 - Angicos - RN - Brazil \\ marianadealbfalcao@gmail.com, raamon.williams@hotmail.com, \{welliana.ramalho,, \\ leilane.gomes\}@ufersa.edu.br
}

Abstract. Requirements engineering enters as an indispensable factor to guarantee the success of softwares, understanding its particularities and contexts. Although classical techniques can be transferred to this scope, it is necessary to analyze and understand the inherent limitations of mobile software. From this demand, the objective of this work is to carry out a study on the techniques of elicitation. With the bibliographic analysis, the techniques will be executed in the software called Pratique - whose purpose is to spread the practice of sports among people. With the results of the study, it is possible to assist in the improvement of the requirements specifications and the development of mobile applications.

Resumo. A engenharia de requisitos entra como um fator indispensável para garantir o sucesso de softwares, entendendo suas particularidades e contextos. Embora as técnicas clássicas possam ser transferidas para esse âmbito, se faz necessário analisar e entender as limitações inerentes dos softwares móveis. A partir dessa demanda o objetivo deste trabalho é realizar um estudo sobre as técnicas de elicitação. Com a análise bibliográfica as técnicas elencadas serão aplicadas no aplicativo Pratique, software cujo objetivo é difundir a prática de esportes entre as pessoas. Com os resultados desse estudo é possível auxiliar na melhoria das especificações de requisitos e no desenvolvimento de aplicativos móveis.

\section{Introdução}

Segundo a Fundação Getúlio Vargas (FGV), em 2017 havia 280 milhões de aparelhos celulares conectados à Internet no Brasil. Tornando o mercado de aplicativos um atrativo importante para novos empreendedores. Muitos desses aplicativos tentam oferecer ao consumidor autonomia no processo de compra de produtos e serviços, no consumo de entretenimento, entre outros. No entanto, além da própria funcionalidade, deve-se ater à qualidade dos aplicativos. Uma forma de garantir o sucesso de um software desde a fase inicial do projeto é elicitar seus requisitos com qualidade, identificando e definindo as estratégias de desenvolvimento do produto ou serviço (JAKKAEW e HONGTHONG, 2017). 
No desenvolvimento de um software, a engenharia de requisitos é um fator fundamental para se alcançar o sucesso de um projeto, uma vez que os maiores problemas na criação de um software estão relacionados à má coleta das informações. Lindstrom (1993) afirma que um requisito mal elicitado ou não elicitado pode causar uma série de insucessos, incluindo a inutilização de sistemas pouco tempo após terem sido implantados. Uma engenharia de requisitos imprecisa pode provocar problemas no projeto de uma aplicação, afetando os custos e prazos envolvidos. Desta forma, projetar sistemas eficientes é de extrema importância para a indústria de software como um todo.

Neste ínterim, a proposta deste trabalho é voltada a sugestão de técnicas de elicitação de requisitos dedicada ao desenvolvimento de softwares para dispositivos móveis. Essa concepção será empregada no desenvolvimento do aplicativo Pratique, como forma de assegurar a qualidade do programa resultante e proporcionar o compartilhamento do conhecimento.

\section{Engenharia de Requisitos}

Segundo Sommerville (2011), a Engenharia de Requisitos é o processo constante elicitação, análise, documentação e verificação de serviços e restrições pertencentes a um sistema. Sem isso, o utilitário final tem grande probabilidade de não atender os anseios do principal interessado (PRESSMAN, 2011).

Entretanto, a fase mais importante no desenvolvimento de um sistema é a elicitação dos requisitos (ZOWGHI, 2002), etapa em que todas as necessidades dos usuários são estudadas e as funcionalidades do sistema são elencadas considerando restrições técnicas, sociais e organizacionais. Sommerville (2011) apresenta um modelo do processo de elicitação e análise de requisitos composto por quatro atividades expostas a seguir:

1. Descoberta de requisitos: nesta fase há a necessidade de interação entre os stakeholders do software para descobrir os requisitos ideais.

2. Classificação e organização de requisitos: nesta atividade são analisados os requisitos coletados para agrupá-los em conjuntos relacionados, organizando-os em grupos coesos.

3. Priorização e negociação de requisitos: considerada uma das fases mais difíceis no processo de elicitação dos requisitos, uma vez que busca priorizar os requisitos identificados e resolver possíveis conflitos entre os stakeholders.

4. Especificação de requisitos: etapa na qual os requisitos serão documentados, podendo ser produzidos documentos formais ou informais.

A elicitação de requisitos não pode ser vista apenas como um problema de dimensão tecnológica, uma vez que nesta atividade o contexto social é mais crítico do que na fase de programação, especificação e projeto. Dessa forma, para haver uma elicitação bem executada, são utilizadas técnicas que ajudam a melhorar a comunicação e resolver eventuais problemas de forma rápida e precisa. Algumas das técnicas mais 
utilizadas são: etnografia, questionário, casos de uso, prototipação, cenários, brainstorming.

\section{Aplicativo Pratique}

O Pratique é um aplicativo que tem como objetivo a difusão da prática esportiva a vários perfis de usuários, conectando-os em uma rede de preferências esportivas, ou seja, o software, além de social, busca fornecer condições que contribuam para o bem físico de seus usuários.

O aplicativo utilizará o recurso de geolocalização que exibirá ao usuário os possíveis locais em que poderá praticar os esportes desejados e modalidades de interesse. $\mathrm{O}$ usuário final também poderá escolher até três modalidades de esportes para praticar e alterar campos como: foto do perfil, idade, modalidades de interesse, preferências de busca de outros usuários. Além de ter acesso ao recurso de chat, onde poderão conversar e marcar atividades coletivas.

\section{Resultados e Discussões}

O desenvolvimento de uma aplicação exige o cumprimento de várias fases iniciais. Uma delas é a escolha da técnica de elicitação de requisitos mais adequada ao contexto da aplicação. Para selecionar os métodos de elicitação deve-se partir do princípio de qual o melhor procedimento que se adapta ao projeto, a equipe de desenvolvimento e a organização (BATISTA e CARVALHO, 2003).

Dessa forma, este trabalho propõe uma síntese dos trabalhos publicados relacionados ao tema elicitação de requisitos, em que centra-se nos seguintes aspectos: tempo de desenvolvimento, qualidade, custo, validação dos requisitos, confiabilidade e experiência do usuário. Para tal, considerou-se como estudo de caso o aplicativo Pratique.

No levantamento dos trabalhos publicados foram considerados os bancos bibliográficos com maior diversidade de publicações: Google Acadêmico, IEEE, SciELO. Foram encontrados 8 trabalhos referiam-se a elicitação de requisitos em dispositivos móveis. Das pesquisas utilizadas como referência, 6 são artigos científicos, 1 é trabalho de conclusão de curso e 1 é dissertação de mestrado.

Com o objetivo de identificar as técnicas de elicitação de requisitos utilizadas em cada trabalho, foi desenvolvida uma Tabela 1. Já para auxiliar na análise das referências bibliográficas selecionadas, foi desenvolvida uma tabela contendo questionamentos sobre atributos que foram considerados em cada trabalho. O resultado dessa etapa é apresentado na Tabela 2. A união dessas tabelas possibilita auxiliar na tomada de decisão de quais técnicas de elicitação de requisitos deverão ser utilizadas no desenvolvimento do aplicativo Pratique.

Considerando as técnicas de elicitação de requisitos, pode-se utilizar ferramentas como a matriz de decisão para analisar e decidir os critérios de importância de parâmetros (PUGH, 1991). A utilização dessa matriz busca validar os requisitos do 
sistema, além de estabelecer quais seriam obrigatórios e desejáveis, como elemento de ponderação.

Segundo Barbosa (2009) a matriz de decisão apresenta linhas que representam características desejáveis ao projeto de software. Já as colunas devem ser constituídas pelas técnicas a serem comparadas. Cada técnica analisada deve receber um peso em relação às características listadas. A partir das técnicas listadas, é atribuída uma nota em referência aos parâmetros selecionados. As notas são valores que indicam o quão desejável é cada característica. Isto posto, as características receberão notas de 0 a 5 fazendo referência às classificações de "Minimização" e "Maximização", respectivamente. Ou seja, os parâmetros com notas de 0 a 2 serão minimizados no projeto e aqueles com notas entre 3 a 5 serão maximizados.

Além disso, a matriz de decisão irá conter pesos atribuídos às técnicas de elicitação de requisitos. Seu valor será referente ao cruzamento das tabelas 1 e 2 , uma vez que ao utilizar as técnicas de elicitação demonstradas na Tabela 1 foram obtidos os resultados expostos na Tabela 2. Os valores dos pesos também variaram entre 0 a 5 e terão a mesma referência de classificação.

A escolha do procedimento encontra-se no maior somatório obtido na matriz de decisão, ou seja, a técnica que obtiver a maior pontuação qualifica-se como a melhor para o projeto. O cálculo dessa pontuação se dá pela multiplicação do peso pela nota atribuída. Estas pontuações serão calculadas por meio da multiplicação do peso pela nota.

Para estabelecer avaliadores na matriz de decisão foi utilizado como base o trabalho de Belgamo e Martins (2000). Dentre os critérios de avaliação apresentados pelo autor, foram selecionados os parâmetros que coincidem com os critérios analisados na Tabela 2. São eles: grupo/indivíduo, confiabilidade, qualidade, custo, tempo e validação.

A escolha das técnicas a serem analisadas na matriz de decisão considera que o aplicativo conta com poucos recursos. Dentre as técnicas possíveis, este estudo limitou-se à utilização das mais aplicadas nas referências estudadas. Foram consideradas: brainstorming, questionário, entrevista, documento de requisitos e prototipagem.

Com o conjunto de técnicas e métricas estabelecidas foi criada a matriz de decisão, que é apresentada na Tabela 3. É possível observar que as técnicas que mais contribuem para o desenvolvimento do software são a prototipagem, o questionário e o documento de requisitos. Após o resultado da matriz de decisão, as técnicas escolhidas foram aplicadas no aplicativo Pratique com o objetivo de identificar os requisitos funcionais e não funcionais, validar as funcionalidades almejadas, entender o público alvo junto às suas preferências e necessidades. 
Tabela 1. Técnicas de elicitação de requisitos utilizadas

\begin{tabular}{|c|c|c|c|c|c|c|c|c|}
\hline \multicolumn{9}{|c|}{ Referência } \\
\hline Técnicas & $\begin{array}{c}\text { (SHAHIDI e } \\
\text { KASIRUN, 2009) }\end{array}$ & $\begin{array}{l}\text { (SEYFF et al., } \\
2010\end{array}$ & $\begin{array}{l}\text { (ROUIBAH e AL- } \\
\text { RAFEE, 2009) }\end{array}$ & $\begin{array}{l}\text { (BARRA, et al, } \\
\text { 2017) }\end{array}$ & (SOUZA, 2014) & $\begin{array}{c}\text { (OSSADA, } \\
\text { MARTINS, } \\
2010)\end{array}$ & $\begin{array}{c}\text { (DANTAS, } \\
\text { 2009) }\end{array}$ & (KELM, 2016) \\
\hline Brainstorming & & $\mathrm{x}$ & & & & & & $\mathrm{x}$ \\
\hline Questionário & & & $\mathrm{x}$ & & & $\mathrm{x}$ & $\mathrm{x}$ & \\
\hline Entrevista & & & $\mathrm{x}$ & & & $\mathrm{x}$ & $\mathrm{x}$ & \\
\hline \multicolumn{9}{|c|}{ Documentação de } \\
\hline Requisitos & & $\mathrm{x}$ & & & & $\mathrm{x}$ & & \\
\hline Prototipação & & $\mathrm{x}$ & & $\mathrm{x}$ & $\mathrm{x}$ & & & $\mathrm{x}$ \\
\hline \multicolumn{9}{|l|}{ Casos De Uso } \\
\hline Etnografia & $\mathrm{x}$ & & & & & & & \\
\hline
\end{tabular}

Tabela 2. Análise comparativa entre as referências utilizadas

\begin{tabular}{|c|c|c|c|c|c|c|c|}
\hline Perguntas & $\begin{array}{l}\text { (SHAHIDI e (SEYFF et al., } 2010 \\
\text { KASIRUN, 2009) }\end{array}$ & $\begin{array}{l}\text { (ROUIBAH e AL- } \\
\text { RAFEE, 2009) }\end{array}$ & $\begin{array}{l}\text { (BARRA, } \\
\text { al., 2017) }\end{array}$ & et (SOUZA, 2014) & $\begin{array}{l}(\text { OSSADA, } \\
\text { MARTINS, 2010) }\end{array}$ & (DANTAS, 2009) & (KELM, 2016) \\
\hline
\end{tabular}

Exigia rapidez na entrega do software?

O desenvolvimento focou na qualidade?

$\mathrm{X}$

$\mathrm{x}$

$\mathrm{x}$

software exigiu limite de

custo?

Os requisitos foran

facilmente validados?

o levantamento de requisitos foi confiável?

O desenvolvimento focou na experiência do usuário? $\mathrm{x}$

$\mathrm{x}$

$\mathrm{x}$

$\mathrm{x}$ $\mathrm{x}$ $\mathrm{x}$ 
Tabela 3. Matriz de decisão

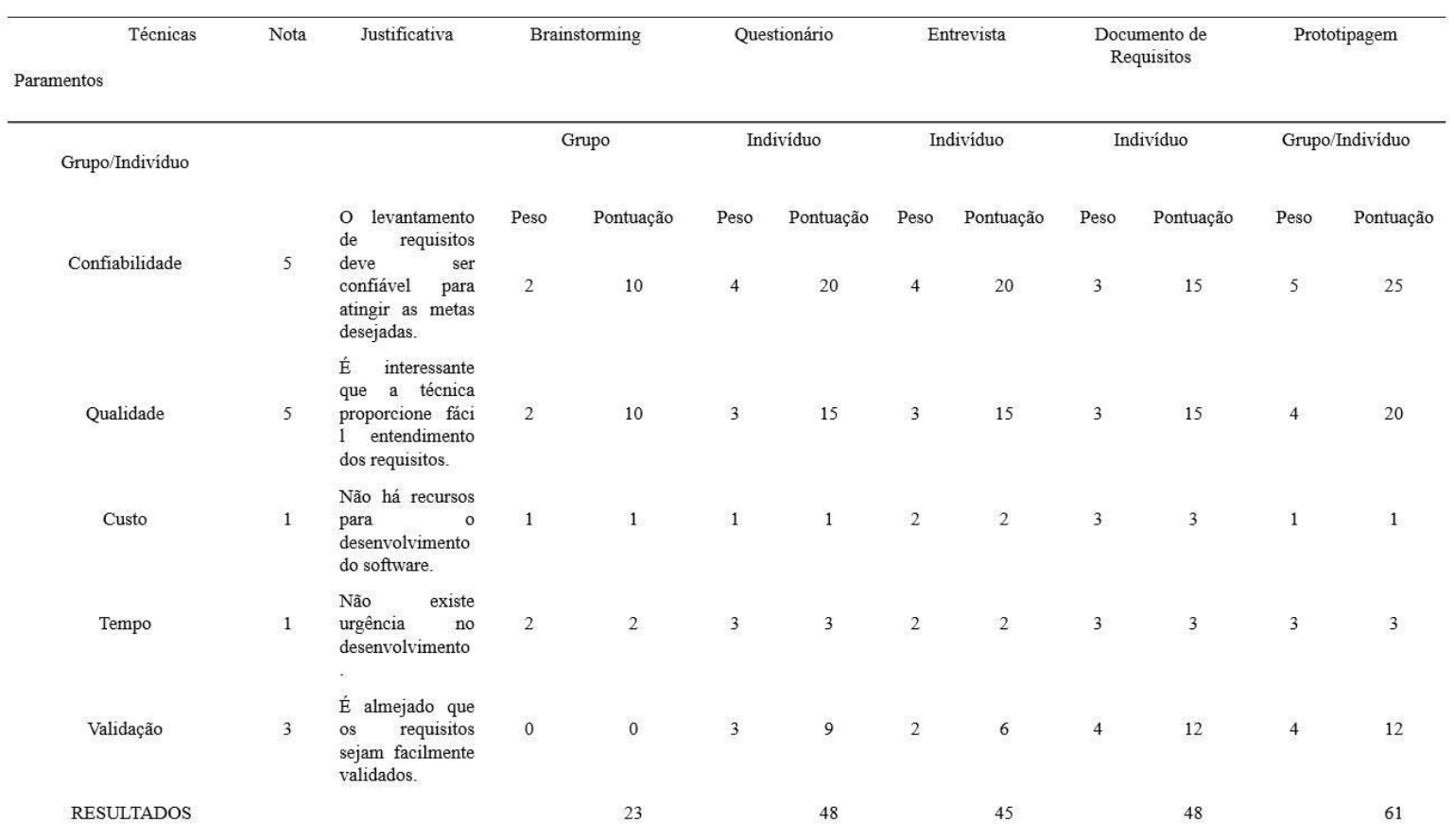

\section{Aplicação das técnicas de engenharia de requisitos para o uso do aplicativo Pratique}

Para auxiliar na determinação das regras de negócio do aplicativo Pratique, além de estabelecer os requisitos iniciais para o desenvolvimento do aplicativo, foi realizado um questionário estruturado na plataforma online Google Forms. O questionário foi aplicado nas cidades de Mossoró/RN e Angicos/RN para identificar quais os esportes mais praticados em cada cidade e investigar os motivos que impedem os entrevistados de praticar atividades esportivas.

Como resultado, obteve-se 73 respostas na cidade de Mossoró. Já o questionário destinado à cidade de Angicos/RN obteve 149 respostas. Nessa pesquisa foi constatado que os entrevistados das duas cidades praticam esportes, sendo o futebol a modalidade mais praticada, seguido de corrida e natação. Para as pessoas que não praticam esportes foi constatado que gostariam de mudar de hábito, e a maioria do público afirmou ter mais interesse em desenvolver atividades ligadas à natação, corrida e vôlei. Além disso, foi perguntado aos usuários qual o principal motivo para não praticar as atividades esportivas, relataram a falta de tempo como principal empecilho, seguido da pouca força de vontade, afirmam também a falta de companheiros esportivos como um problema.

Com esses dados foi possível identificar as modalidades esportivas mais praticadas e mais desejadas pelos entrevistados. Dessa forma, o aplicativo Pratique vai ser desenvolvido inicialmente com enfoque nas seguintes modalidades esportivas: natação, vôlei, futebol, futsal, corrida e musculação. 
Após a aplicação do questionário a próxima etapa do processo de elicitação de requisitos foi delimitar o escopo do conjunto de funcionalidades. A elaboração do documento deve acontecer de forma concisa, objetiva e clara, principalmente aos interessados na aplicação como forma de evitar problemas de ambiguidade.

Como a principal finalidade da documentação de requisitos está na definição do escopo, este documento encontra-se estruturado em: Introdução, visão geral do documento, descrição dos requisitos funcionais e não funcionais e casos de uso. A modelagem de casos de uso foi desenvolvida a partir da descrição dos requisitos usando a ferramenta Astah, versão 7.1. Parte do documento de requisitos encontra-se a seguir:

\section{Requisitos do Software}

Requisitos Funcionais

Os requisitos que descrevem os aspectos funcionais do sistema são apresentados a seguir:

Requisitos de Segurança

\begin{tabular}{|l|l|}
\hline Ident. & Descrição \\
\hline RF/SEG-01 & Todo usuário para ter acesso ao aplicativo efetuará um login e senha para a ativação da sua conta. \\
\hline RF/SEG-02 & Todo o cliente para ter acesso ao aplicativo efetuará um login e senha para a ativação da sua conta. \\
\hline
\end{tabular}

Requisitos de Operacionais

\begin{tabular}{|c|c|}
\hline Ident. & Descrição \\
\hline RF/OPE-01 & $\begin{array}{l}\text { A partir da geolocalização, , ferramenta que auxilia a busca para o usuário os possíveis locais em que poderá ser } \\
\text { praticado os esportes desejados e modalidades de interesse, públicos ou provados. }\end{array}$ \\
\hline RF/OPE-02 & $\begin{array}{l}\text { A partir da geolocalização será exibido ao usuário os possíveis locais em que poderá ser comprado os materiais } \\
\text { esportivos desejados por ele. }\end{array}$ \\
\hline RF/OPE-03 & O usuário final poderá escolher até três modalidades de esportes para ser praticado ao se cadastrar no aplicativo. \\
\hline RF/OPE-04 & O usuário do aplicativo terá o recurso de buscar outros usuários por username na aplicação. \\
\hline RF/OPE-05 & O usuário poderá adicionar outro usuário como amigo. \\
\hline RF/OPE-06 & O usuário poderá conversar com outros integrantes do aplicativo. \\
\hline RF/OPE-07 & O usuário poderá excluir outro usuário do seu círculo de amigos. \\
\hline RF/OPE-O8 & O usuário poderá bloquear outros usuários. \\
\hline RF/OPE-09 & O usuário poderá mandar mensagens para os clientes, donos de quadras esportivas ou de suas lojas esportivas. \\
\hline RF/OPE-10 & O cliente poderá responder o usuário através de um recurso de chat incorporado no sistema. \\
\hline$R F / O P E-11$ & O cliente poderá mandar notificações com promoções dos seus produtos. \\
\hline$R F / O P E-12$ & O cliente poderá atualizar suas próprias informações do perfil. \\
\hline$R F / O P E-13$ & A aplicação contará com a portabilidade para a plataforma móvel. \\
\hline RF/OPE-14 & O usuário poderá avaliar amigos com intuito de dar maior segurança aos demais. \\
\hline RF/OPE-15 & O usuário poderá criar partidas dentro do aplicativo. \\
\hline
\end{tabular}


Requisitos de Segurança

\begin{tabular}{|l|l|}
\hline Ident. & Descrição \\
\hline RNF/SEG-01 & O aplicativo precisa guardar as informações dos usuários finais com segurança. \\
\hline RNF/SEG-02 & O aplicativo precisa guardar as informações dos clientes com segurança. \\
\hline
\end{tabular}

Requisitos de Interface

\begin{tabular}{|l|l|}
\hline Ident. & Descrição \\
\hline RNF/INT-01 & O sistema deve ser atrativo ao usuário, de fácil aprendizado e recordação \\
\hline
\end{tabular}

Requisitos de Operacionais

\begin{tabular}{|l|l|}
\hline Ident. & Descrição \\
\hline$R N F / O P E-01$ & O sistema deve ser desenvolvido em Java para Android. \\
\hline RNF/OPE-02 & O sistema deve atender as normas de usabilidade segundo a ISO/IEC 9126 \\
\hline
\end{tabular}

Requisitos de Confiabilidade

\begin{tabular}{|l|l|}
\hline Ident. & Descrição \\
\hline RNF/CON-01 & $\begin{array}{l}\text { O sistema deve estar disponível } 24 \text { horas por dia durante os } 7 \text { dias da semana. Por não se tratar de um sistema crítico, o } \\
\text { sistema poderá ficar fora do ar até que seja corrigida alguma falha que possa ocorrer. }\end{array}$ \\
\hline
\end{tabular}

A norma ISO/IEC 9126 foi utilizada como referência para o cumprimento desta etapa com eficiência. Segundo essa norma, para um software ser desenvolvido com qualidade ele deve ser analisado sob algumas sub características. São elas: funcionalidade, usabilidade, eficiência e portabilidade. Os requisitos não funcionais contêm limitações do produto, e foram analisados de acordo com a norma, observando os fatores de usabilidade, eficiência e confiabilidade. Juntamente com o desenvolvimento do documento de requisitos foi alcançado a identificação e negociação das funcionalidades. Os requisitos darão apoio à criação de protótipos, minimizando erros e inconformidades.

Porém, por mais que a documentação garanta informações contextuais no aplicativo, levantando um grande volume de informações, é durante a interação do usuário com o aplicativo que os detalhes serão percebidos. Em razão disso, foram criados protótipos de baixa e alta fidelidade, um modelo semi funcional do sistema que simula as ações e preferências de usuários.

A utilização de protótipos de baixa fidelidade, apresentados na Figura 1, teve como principal benefício apresentar a arquitetura da informação, no qual foi apresentado um layout do sistema e abstraído onde as funcionalidades idealizadas para o aplicativo irão ficar. Além disso, esses protótipos foram fundamentais para avaliar algumas soluções na fase inicial do desenvolvimento. Já os protótipos de alta fidelidade, 
descritos na Figura 2, foram criados principalmente para atingir um maior grau de detalhamento de funcionalidades, além do refinamento estético. A metodologia do protótipo escolhida foi a prototipação evolucionária, em virtude da produção de um modelo inicial e o refinamento ao longo das várias fases de desenvolvimento.
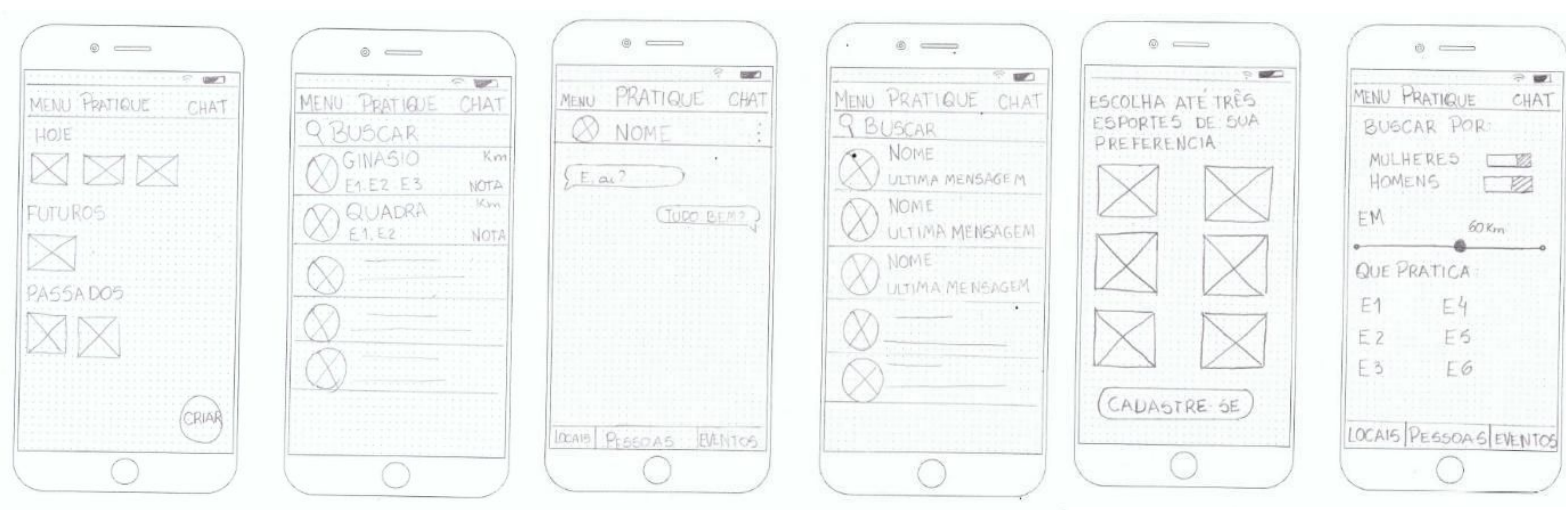

Figura 1. Protótipos de baixa fidelidade do aplicativo Pratique

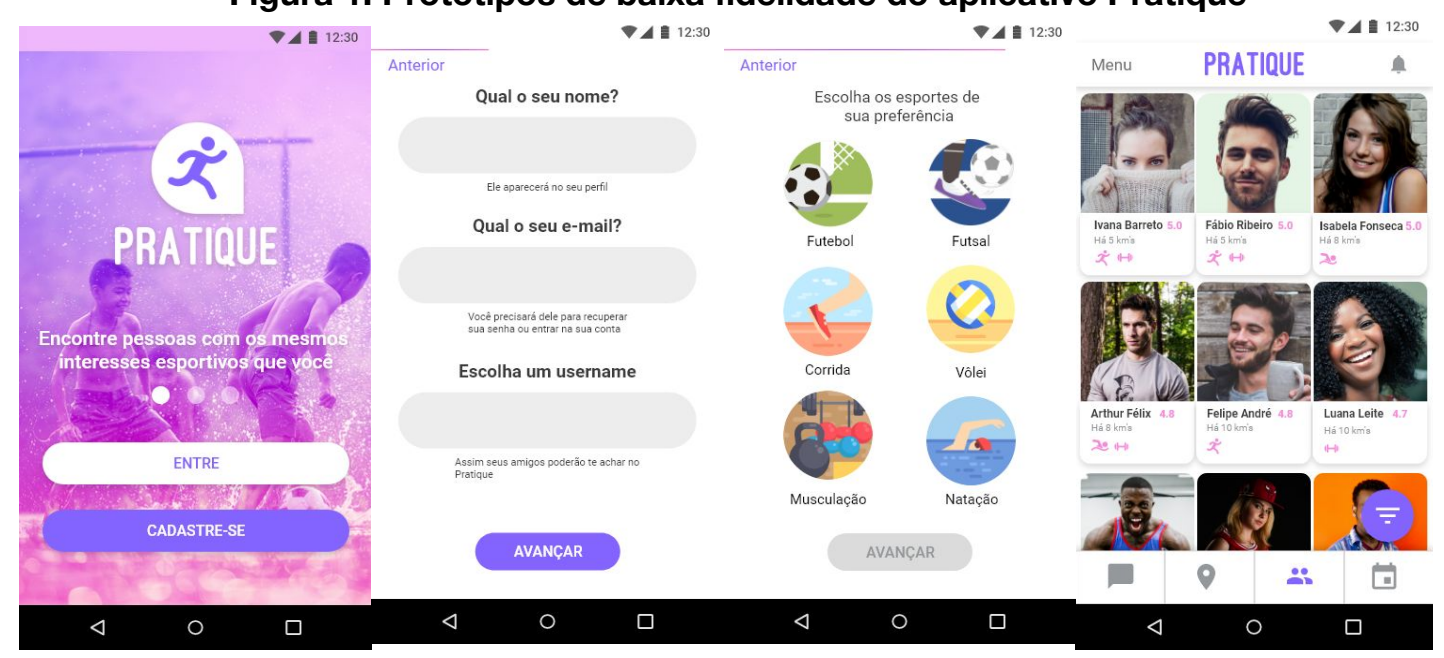

Figura 2. Protótipos de alta fidelidade do aplicativo Pratique

\section{Conclusão}

O propósito inicial deste trabalho foi estudar as técnicas de elicitação de requisitos voltadas para os dispositivos móveis. Após a análise bibliográfica foi realizado uma matriz de decisão para apoiar as escolhas das técnicas a serem utilizadas no aplicativo Pratique.

Como resultado da matriz de decisão foi obtido que as técnicas ideais para o aplicativo Pratique, são: questionario, documento de requisitos e prototipação. Cada técnica possibilitou a coleta de requisitos de forma ágil e concisa. Além disso, cada etapa de coleta de requisitos contribuiu para decisões funcionais do sistema a ser implementado. 
Com a realização das etapas descritas acima é possível que o aplicativo Pratique consiga atingir os parâmetros necessários para realização de uma boa elicitação de requisitos, contribuindo para o sucesso da aplicação. Assim, os objetivos do trabalho foram satisfatórios, gerando conhecimento como forma de contribuir com novos conhecimentos na área.

\section{Referencias}

Barbosa, Gl. et al. (2009) "Um processo de Elicitação de Requisitos com foco na seleção da Técnica de Elicitação", VIII Simpósio Brasileiro de Qualidade de Software, p. 159-173.

Batista, E. A.; Carvalho, A. M. B. R. (2003) "Uma Taxonomia Facetada para Técnicas de Elicitação de Requisitos”, In: WER p. 48-62.

Belgamo, A.; Martins, L. E. G. (2000) "Estudo comparativo sobre as técnicas de elicitação de requisitos do software", In: XX Congresso Brasileiro da Sociedade Brasileira de Computação (SBC), Curitiba-Paraná.

Ceará, A. L. do E. do. (2014) "Internautas apontam falta de investimento no esporte brasileiro.", $<$ https://alce.jusbrasil.com.br/noticias/100028066/internautas-apontam-fa lta-de-investimento-no-esporte-brasileiro> , Setembro.Jakkaew, P.; HONGTHONG, T. (2017) Requirements elicitation to develop mobile application for elderly.", In: Digital Arts, Media and Technology (ICDAMT), International Conference on. IEEE. p. 464-467.

Lindstrom, D. R. Five ways to destroy a development project (software development)", IEEE Software, v. 10, n. 5, p. 55-58, 1993.

Presman, R. S. (2011) "Engenharia de Software”, Rio de Janeiro, Makron Books. p. 780.

Pugh, S. (1990,1991) "Total design: integrated methods for successful product engineering."

Sommerville, I. (2011) “Engenharia de Software.”, tradução Ivan Bosnic e Kalink G. de O. Gonçalves, revisão técnica Kechi Hirama, 9.ed.,São Paulo,Pearson Prentice Hall. Título Original: Software Engineering. .

Thayer, R. H.; Dorfman, M. (1997) Introduction to Tutorial Software Requirements Enginnering. Software Requirements Engineering, IEEE-CS Press,, p. 1-2.

Vargas, F. G. "Tecnologia de Informação", São Paulo: 28a Pesquisa Anual do Uso de Ti, 2017. 24 slides, color.

Zowghi, D. (2002) "Does global software development need a different requirements engineering process", In: Proceedings of International Workshop on Global Software Development.. 